\title{
Orthopairs and granular computing
}

\author{
${\text { Davide } \text { Ciucci }^{1} \text { (I) }}$
}

Received: 31 August 2015/Accepted: 19 December 2015/Published online: 1 February 2016

(C) Springer International Publishing Switzerland 2016

\begin{abstract}
Pairs of disjoint sets (orthopairs) naturally arise or have points in common with many tools to manage uncertainty: rough sets, shadowed sets, version spaces, three-valued logics, etc. Indeed, they can be used to model partial knowledge, borderline cases, consensus, examples and counter-examples pairs. Moreover, generalized versions of orthopairs are the well known theories of Atanassov intuitionistic fuzzy sets and possibility theory and the newly established three-way decision theory. Thus, it is worth studying them on an abstract level in order to outline general properties that can then be casted to the different paradigms they are in connection with. In this paper, we will review how to define orthopairs and a hierarchy on them in the light of granular computing. Aggregation operators will also be discussed as well as possible generalizations and connections with different paradigms. This will permit us to point out new facets of these paradigms and outline some possible future developments.
\end{abstract}

Keywords Orthopair $\cdot$ Partial knowledge $\cdot$ Rough sets

\section{Introduction}

An orthopair is a pair of disjoint subsets of a given universe $X$, we denote it as $O=(P, N)$. The two letters $P$ and $N$ directly points to the semantics usually attached to an orthopair: $P$ standing for positive and $N$ for negative. Indeed, an orthopair can be used to collect positive and negative

Davide Ciucci

ciucci@disco.unimib.it

1 DISCo, University of Milano-Bicocca, Viale Sarca 336/14, 20126 Milano, Italy examples, affirmed or negated propositional variables, trust and distrust statements, accepted and rejected objects and so on. An orthopair tri-partitions the universe in three subsets $P, N,(P \cup N)^{c}$, we denote this last term as boundary: Bnd : $=(P \cup N)^{c}$ being the set of unknown objects in a partial knowledge setting. Clearly any combination of these three subsets defines an orthopair. That is, also $(P, B n d)$ and $(B n d, N)$ are orthopairs, even if these two interpretations are rare. On the other hand, it is frequent to find a representation of an orthopair through a nested pair of sets, such as $\left(P, N^{c}\right)$. The set $N^{c}$ represents the non-negative or possibility zone, we will denote it as $U p p$, following the usual rough set nomenclature of Upper approximation (as opposite to the lower approximation represented by $P$ ).

Orthopairs are strictly related to three-valued sets, being possible to establish a bijection between the two notions. Indeed, a three-valued set: $f: X \mapsto\left\{0, \frac{1}{2}, 1\right\}$ corresponds to an orthopair $O$ defined pointwisely for all $x$ as:

$$
\begin{array}{lll}
x \in P & \text { iff } & \mathrm{f}(\mathrm{x})=1 \\
x \in N & \text { iff } & f(\mathrm{x})=0
\end{array}
$$

Thus, all notions on three-valued sets and three-valued logics can be inherited by orthopairs (Ciucci and Dubois 2014; Ciucci et al. 2014). This will be particularly useful when defining operations on orthopairs.

Thanks to their simplicity in representing bipolar information, orthopairs can be found at work in several contexts such as, to make some recent example, trust and distrust statement expressed by experts in Social Network Analysis (Wu et al. 2015), representing partial knowledge (Aguirre et al. 2014) or vague knowledge (Crosscombe and Lawry 2015; Vetterlein 2015), formal concept analysis (Qi et al. 2014).

Pairs of nested or disjoint sets have been studied also in the past. In Ciucci (2014), we reviewed some works made 
in the ' 60 s by Fadini, Andreoli and Gentilhomme (some of their results will also be reported in this paper). More recently, Yao investigated pairs of nested sets under the name interval sets, as a tool to represent imprecise or partial knowledge (Yao 1993, 2009a). In this sense, they are strictly related to orthopairs and all the considerations put forward here could apply also to interval sets and to interval set applications such as clustering (Yao et al. 2009), at least from a purely theoretical standpoint. The difference with respect to our approach lies in the semantics. Interval sets are conceived as a generalization to set of interval arithmetics and they are not considered as threevalued structures but as a "family of sets" (Yao 2009a).

In the present work we will survey orthopairs in the light of granular computing. In particular, there are two key elements in granular computing: granulation of the universe and hierarchy on granules (Yao et al. 2013). Our granules will be orthopairs on a universe $X$. In Sect. 2 we will see some possible ways to define an orthopair, thus how to granulate our universe. To define a hierarchy, we will present in Sect. 3 some ways to define an order relation among orthopairs. Clearly, this naturally defines different levels of granules. The possibility to move along this hierarchy can be given by union and intersection of orthopairs, which are studied in Sect. 4 together with other aggregation operators. Sections 5 and 6 are devoted to present the relationship of orthopairs with some other existing paradigms, which are generalization of orthopairs or based, as orthopairs, on a tri-partition of the universe. Finally, some conclusions are provided and open questions discussed. We notice that here we present a general setting. According to the application we have to handle, we need to specialize and tune the way to define and manage orthopairs.

\section{Granulation}

An orthopair can be seen as a complex granule, made of two sets that can assume different meanings and can be obtained in different ways. We are now going to see some ways to obtain orthopairs (or equivalently nested pairs).

\subsection{Rough Sets}

Rough Set Theory was conceived by Z. Pawlak to manage uncertain information in classification tasks (Pawlak 1982). In more than 30 years of research, it reached a consensus in many applications and it has been generalized in several directions and hybridized with different paradigms, fuzzy sets among all. For a recent overview see the chapters devoted to Rough Set Theory in Kacprzyk and Pedrycz (2015). For the scope of the present work, let us introduce and discuss generalized rough sets based on binary relations. Similar considerations can be, however, put forward for other rough set models.

Definition 1 An approximation space is a pair $(X, \mathcal{R})$ with $X$ a set of objects and $\mathcal{R}$ a binary relation on $X$. The granule generated by $\mathcal{R}$ for a given object $x$ is the set $g_{\mathcal{R}}(x):=\{y \in X: x \mathcal{R} y\}$.

Remark 1 Typically, in rough-set applications, objects are described using some properties and organized in so called Information Tables. Then, the relation $\mathcal{R}$ is obtained looking at the properties of objects: for instance the typical way to consider two objects as equivalent is to check if they assume the same value for all the properties under investigation.

Thus, the minimal unit of information, the granule, is the set of objects reachable through a relation $\mathcal{R}$ from an object $x$ and so, it collects the objects connected to $x$, where the kind of connection depends on $R$ : it can be equivalence (as in the original Pawlak case), similarity, preference, etc. The objects in a granule are considered indiscernible, thus meaning that we cannot single out all the objects and have a perfect knowledge of all the universe. However, given a subset of objects, we can define its approximation.

Definition 2 Let $(X, \mathcal{R})$ be an approximation space. The lower approximation of $H \subseteq X$ is

$l(H):=\left\{x \in X \mid g_{\mathcal{R}}(x) \subseteq H\right\}$

and the upper approximation of $H$ is

$u(H):=\left\{x \in X \mid g_{\mathcal{R}}(x) \cap H \neq \emptyset\right\}$

If the relation is serial, ${ }^{1}$ then $l(H) \subseteq u(H)$ Yao (1998) and the nested pair $(l(H), u(H))$ is named a rough set. The corresponding orthopair $\left(l(H), u^{c}(H)\right)$ also defines the same rough set through the lower approximation and the so called exterior region, denoted as $e(H):=u^{c}(H)$. Finally, the uncertainty region $B n d(H)=u(H) \backslash l(H)$ is the boundary region. Of course also the pair $(l(H), \operatorname{Bnd}(H))$ is an orthopair, even if this notation is less used than the previous one.

The lower approximation of a set $H$ is interpreted as certainty: it contains the objects that certainly belong to $H$, whereas the upper approximation represents the possibility, i.e., it contains the elements that possibly belong to $H$. Of course the exterior $e(H)$ represents the impossibility, that is, the objects that certainly do not belong to $H$.

We remark that in general not all nested/ortho pairs on $X$ can be obtained as approximations of a subset $H$ of the

\footnotetext{
${ }^{1}$ A relation is serial iff any element is in relation through $\mathcal{R}$ with at least one other element. Formally, $\forall x \in X, \exists y \in X: x \mathcal{R} y$.
} 
universe $X$. That is rough sets on an approximation space $(X, R)$ are just a subset of all the orthopairs of $X$. Even in the simpler case of an equivalence relation, this poses some problems when introducing operations on rough sets, since it is not assured that even if the result is a nested (ortho) pair it is also a rough approximation (Ciucci and Dubois 2014).

\subsection{Shadowed sets}

To simplify the infinite precision of fuzzy subsets of a universe $X$, Pedrycz introduced shadowed sets as, basically, a three-valued entity on the same universe $X$ Pedrycz (1998): "shadowed sets are information granules induced by fuzzy sets so that they capture the essence of fuzzy sets at the same time reducing the numeric burden" (Pedrycz 2009). More formally, we can define them as follows.

Definition 3 Let $X$ be a set of objects, called the universe. A shadowed set on $X$ is any mapping $s: X \rightarrow\{0,(0,1), 1\}$.

That is objects are classified in three regions: the core, $s(x)=1$, the region of exclusion, $s(x)=0$ and the shadow, objects we are not able to correctly classify. As can be seen, this is exactly a three-valued set which originates an orthopair.

So, we can say that shadowed sets gives a granulation of the universe in terms of orthopairs, starting from a fuzzy set. This procedure is done through $\alpha$-cuts. That is, we fix a value $\alpha \in\left(0, \frac{1}{2}\right)$, and given the membership function of a fuzzy set $f: X \mapsto[0,1]$ the induced shadowed set is defined as

$s(x):= \begin{cases}0 & \text { if } \mathrm{f}(\mathrm{x}) \leq \alpha \\ 1 & \text { if } \mathrm{f}(\mathrm{x}) \geq 1-\alpha \\ (0,1) & \text { otherwise }\end{cases}$

One of the main characteristic of shadowed sets is the procedure to define the threshold $\alpha$. The aim is to have a balance of uncertainty (Pedrycz and Vukovich 2002; Pedrycz 2005), that is to compensate the change of lower and upper values of $f(x)$ with the values $f(x)$ in the shadow. For any given shape of fuzzy sets, we obtain a typical threshold, for instance in case of a triangular membership function, the optimal value is $\alpha=\sqrt{2}-1$.

\subsection{Partial logical valuations}

Let us consider a set of Boolean variables $\mathcal{A}$ which represent some knowledge on a given domain. Their value or truth assignment can be obtained from the information contained in a relational database or be given by an agent, for instance an expert of the domain. Some information can be missing, so that we are not able to give a truth value to all variables, which are then unknown, giving rise to a partial assignment typical of partial logics Blamey (1985).
Clearly, a partial assignment $v$ gives rise to an orthopair of true-false variables: $P=\{a \in \mathcal{A}: v(a)=1\} \quad$ and $N=\{a \in \mathcal{A}: v(a)=0\}$. In case of complete assignments we get the simple orthopair $\left(P, P^{c}\right)$.

A different but equivalent way to represent such an orthopair is given by consistent Boolean valuation pairs (Lawry and González Rodríguez 2011), that is pairs $(\underline{v}, \bar{v})$ of Boolean valuations on $\{0,1\}$, such that $\underline{v} \leq \bar{v}$ holds pointwisely. The bijection between orthopairs and consistent Boolean valuation pairs is defined as follows

$$
\begin{array}{rll}
a \in P & \text { iff } & \underline{\mathrm{v}}(\mathrm{a})=\overline{\mathrm{v}}(\mathrm{a})=1 \\
a \in N & \text { iff } & \underline{\mathrm{v}}(\mathrm{a})=\overline{\mathrm{v}}(\mathrm{a})=0 \\
a \in(P \cup N)^{c} & \text { iff } & \underline{\mathrm{v}}(\mathrm{a})=0, \overline{\mathrm{v}}(\mathrm{a})=1
\end{array}
$$

We also notice that any orthopair of variables defines a particular possibility distribution (or epistemic set). Indeed, we can collect all the (complete) assignments that are compatible with a (partial) orthopair, that is, the possibility distribution associated to an orthopair $(P, N)$ is the set $\{v: \mathcal{A} \longmapsto\{0,1\}: \forall a \in P, v(a)=1 ; \forall a \in N, v(a)=0\}$.

The other way round is not true, that is not all possibility distributions give rise to an orthopair. We will further discuss this issue in Sect. 5. More discussion on the logical treatment of orthopairs and its different interpretations can be found in Ciucci et al. (2014).

\section{Hierarchy of levels}

The straightforward way to define levels and hierarchy of orthopairs is to define an order relation on them. There are different orders that give rise to lattice structures or simply posets. We are now going to revise these order relations. Let us notice that from a partial order relation on three values we can define an order relation on three valued functions, in a straightforward pointwise way, and then on orthopairs. The other way does not hold in general. That is, not pointwise order relations cannot be defined on three values, but they can be meaningful in orthopairs as we will see.

In Table 1, we give an overview of the (pointwise) orderings we will discuss. We notice that dual orderings

Table 1 A summary of the pointwise order relations on orthopairs

\begin{tabular}{ll}
\hline Order on $\mathbf{3}$ & Order on $\mathbf{O}$ \\
\hline $0 \leq \frac{1}{2} \leq 1$ & $P_{1} \subseteq P_{2}, N_{2} \subseteq N_{1}$ \\
$\frac{1}{2} \leq 1 \leq 0$ & $N_{1} \subseteq N_{2}$, Bnd $_{2} \subseteq B n d_{1}$ \\
$\frac{1}{2} \leq 0 \leq 1$ & $P_{1} \subseteq P_{2}$, Bnd $_{2} \subseteq B n d_{1}$ \\
$\frac{1}{2} \leq 1, \frac{1}{2} \leq 0$ & $P_{1} \subseteq P_{2}, N_{1} \subseteq N_{2}$ \\
$0 \leq \frac{1}{2}, 0 \leq 1$ & $P_{1} \subseteq P_{2}$, Bnd $_{1} \subseteq$ Bnd $_{2}$ \\
$1 \leq \frac{1}{2}, 1 \leq 0$ & $N_{1} \subseteq N_{2}$, Bnd $_{1} \subseteq$ Bnd $_{2}$ \\
\hline
\end{tabular}


with respect to these ones can also be given and studied, i.e., for any order $\preceq$ we can also consider the dual $\preceq_{d}$ defined as $O_{1} \preceq_{d} O_{2}$ iff $O_{2} \preceq O_{1}$. The dual order relations are not listed in Table 1 .

All these order relations produce a different hierarchy, i.e, a different granulation of the universe. We can navigate in these hierarchies by zooming in or zooming out, using the intersection and union operation generated by the ordering.

\subsection{Total orderings}

We have three total order relations (plus the dual ones). At first, the standard order $\preceq_{t}$ on three values and on orthopairs is known as the truth ordering (Belnap 1977) (also proper (Fadini 1962b) or normal inclusion Gentilhomme (1968)):

$$
\begin{aligned}
& x \preceq_{t} y \quad \text { iff } \quad 0 \prec \frac{1}{2} \prec 1 \\
& O_{1} \preceq_{t} O_{2} \quad \text { iff } \quad \mathrm{P}_{1} \subseteq \mathrm{P}_{2}, \mathrm{~N}_{2} \subseteq \mathrm{N}_{1}
\end{aligned}
$$

The fact that an orthopair $O_{2}$ is greater than another one $O_{1}$ is interpreted as if $O_{2}$ is "more true" than $O_{1}$, since it has more positive elements and less negative ones than $O_{1}$.

Example 1 Let us consider a granulation of the universe given by partial evaluations (see Sect. 2.3). Then, the hierarchy built by this ordering has as a top element the orthopair corresponding to assigning true to all variables and as bottom element the orthopairs with all variables set to false. A path from top to bottom, means diminishing true assignments and augmenting false ones (with some that can be unknown). For instance, in case of four variables $\{a, b, c, d\}$, we have $(\emptyset,\{a, b, c, d\}) \preceq_{t}(\{a\},\{b, c, d\}) \preceq_{t}$ $(\{a\},\{c, d\}) \preceq_{t}(\{a, b, c, d\}, \emptyset)$.

The other two orderings are named "one-sided information" ordering. The term "one sided" is due to the fact that positive and negative part do not play the same role: in the case of $\preceq_{N}$ the negative information is preserved whereas in $\preceq_{P}$ the positive one is. On orthopairs they read as:

$$
\begin{array}{lll}
O_{1} \preceq_{N} O_{2} & \text { iff } & \mathrm{N}_{1} \subseteq \mathrm{N}_{2}, \mathrm{Bnd}_{2} \subseteq \mathrm{Bnd}_{1} \\
O_{1} \preceq_{P} O_{2} & \text { iff } & \mathrm{P}_{1} \subseteq \mathrm{P}_{2}, \mathrm{Bnd}_{2} \subseteq \mathrm{Bnd}_{1}
\end{array}
$$

and the corresponding orders on three values are

$$
\begin{array}{ll}
x \preceq_{N} y \quad \text { iff } \quad \frac{1}{2} \prec 1 \prec 0 \\
x \preceq_{P} y \quad \text { iff } \quad \frac{1}{2} \prec 0 \prec 1
\end{array}
$$

We remark that all these three order relations give rise to a lattice structure whose meet and join will be given in Sect. 4.1 .

\subsection{Partial orderings}

The most important partial order relation we are going to introduce has received attention in several fields, and it is known as knowledge ordering (Belnap 1977; Yao 2009a) or semantic precision (Lawry and Dubois 2012) or sharpening relation (Shapiro 2006). We will use the symbol $\preceq_{I}$ to denote it, the $I$ standing for information:

$$
O_{1} \preceq_{I} O_{2} \quad \text { iff } \quad \mathrm{P}_{1} \subseteq \mathrm{P}_{2}, \mathrm{~N}_{1} \subseteq \mathrm{N}_{2}
$$

We can interpret it as the orthopair $O_{1}$ being less informative than the orthopair $\mathrm{O}_{2}$, since the uncertainty region is wider and this is due to a greater knowledge in both positive and negative parts. This contrasts with the previous introduced orders $\preceq_{N}$ and $\preceq_{P}$ where the difference in the boundary was due only to one of the two sides. The formal relationships among these three orders is:

$$
\begin{aligned}
& \left(P_{1}, N_{1}\right) \preceq_{I}\left(P_{2}, N_{2}\right) \Longleftrightarrow\left(P_{1}, N_{1}\right) \preceq_{N}\left(P_{2}, N_{2}\right) \\
& \quad \text { and } \quad\left(P_{1}, N_{1}\right) \preceq_{P}\left(P_{2}, N_{2}\right) .
\end{aligned}
$$

That is the information ordering implies the two one-sided orders and we need both the one sided to get the information ordering.

Example 2 Let us consider a granulation based on rough sets. This ordering (which is not the standard one used in rough set theory, which is $\preceq_{t}$ ) can be used to represent an increase of knowledge. Indeed, if we add an attribute to characterize our objects (i.e., we acquire more knowledge, for instance making a further diagnostic test in medicine), then the granulation of the universe becomes finer and our orthopairs can be ordered with $\preceq_{I}$.

On three values, it corresponds to the partial order where $\frac{1}{2}$ is less than both 0 and 1 (the unknown is less informative than the known values true and false):

$x \preceq_{I} y \quad$ iff $\quad \frac{1}{2} \prec 0, \frac{1}{2} \prec 1$

Of course, this order gives rise only to a meet semi-lattice.

The other two partial order relations move information from the positive (negative) side of $O_{1}$ to the negative (positive) side or to the boundary of $\mathrm{O}_{2}$ with the boundary of $\mathrm{O}_{2}$ being greater. That is, there is more uncertainty in $\mathrm{O}_{2}$ than in $O_{1}$. The first one reads $0 \leq \frac{1}{2}, 0 \leq 1$ on three values. On orthopairs it becomes

$$
P_{1} \subseteq P_{2} \quad \text { and } \quad \mathrm{Bnd}_{1} \subseteq \mathrm{Bnd}_{2} .
$$

In this case, $\mathrm{O}_{2}$ has less negative knowledge: some negative points in $N_{1}$ are moved to $P_{2}$, thus becoming positive and some others to $B n d_{2}$. So, the second orthopair from negative becomes possibilistic with respect to these last points. 
The last partial order relation is given by $1 \leq \frac{1}{2}, 1 \leq 2$ and on orthopairs by

$N_{1} \subseteq N_{2}$ and $\quad$ Bnd $_{1} \subseteq$ Bnd $_{2}$

and it acts in the opposite direction: some objects in $P_{1}$ move to the negative side or to the boundary in $\mathrm{O}_{2}$. So, $\mathrm{O}_{2}$ is greater than $O_{1}$ can be interpreted as $O_{2}$ is more negative or pessimistic than $O_{1}$.

Both these order relations define a meet semi-lattice.

\subsection{Non pointwise orderings}

These kind of order relations were investigated by Fadini Fadini (1962b) and they are greatly influenced by his view of an orthopair as (positive, boundary). Indeed, positive and boundary regions play a major role in defining these orderings. The essential condition required is that $P_{1} \cup B n d_{1} \subseteq P_{2} \cup B n d_{2}$, which is equivalent to $N_{2} \subseteq N_{1}$, that is the negative knowledge is less in the greater element $\mathrm{O}_{2}$. Apart from the information ordering (7), he defines four other situations that satisfy this constraint. They are:

(npo1-neg) Total inclusion: $\left(P_{1} \cup B n d_{1}\right) \subseteq P_{2}$, the name is clearly given by the intuition that the orthopair $O_{1}$ it totally contained in $\mathrm{O}_{2}$, both in its certainty and possibility parts. This ordering has also been named strong in Gentilhomme (1968) with respect to the weak one: $P_{1} \subseteq P_{2} \cup$ Bnd $_{2}$.

(npo2-neg) Improper or inverted inclusion: $B n d_{1} \subseteq P_{2}, P_{1} \subseteq B n d_{2}$. Everything that is positive in $O_{1}$ becomes uncertain in $\mathrm{O}_{2}$ and everything that is uncertain become positive.

(npo3-neg) Total improper inclusion: $P_{1} \cup B n d_{1} \subseteq B n d_{2}$, everything that is positive in $O_{1}$ becomes uncertain.

(npo4-neg) Mixed inclusion: $B n d_{1}$ and $P_{1}$ are both contained in $P_{2} \cup B n d_{2}$ and they have a non-empty intersection with both $P_{2}$ and $B n d_{2}$.

Clearly, if we change the constraint $N_{2} \subseteq N_{1}$ with the corresponding positive condition $P_{1} \subseteq P_{2}$, other similar orders could be defined:

(npo1-pos) $\quad N_{2} \cup \mathrm{Bnd}_{2} \subseteq N_{1}$ : not only $\mathrm{O}_{2}$ has more knowledge than $O_{1}$ but this is due to the fact that the uncertainty in $\mathrm{O}_{2}$ is all due to some negative information that becomes uncertain.

(npo2-pos) Bnd $d_{2} \subseteq N_{1}, N_{2} \subseteq B n d_{1}$. Everything that is negative in $O_{2}$ was uncertain in $O_{1}$ and everything that is uncertain was negative.

(npo3-pos) $\quad N_{2} \cup B n d_{2} \subseteq B n d_{1}$, everything that was negative or uncertain in $O_{1}$ becomes uncertain in $O_{2}$. This also implies that $N_{1} \subseteq P_{2}$, that is all the negative information becomes positive.
Fig. 1 Three levels of an orthopair of alleles

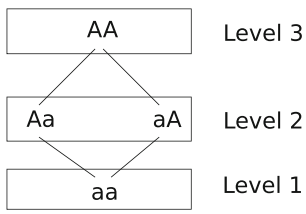

\subsection{An orthopair as a hierarchy}

A different approach with respect to the idea of an orthopairs as a granule and then levels made of orthopairs, is to consider a single orthopair as a structure itself. In this case we have only three levels and granules are simple objects. This idea was put forward by Andreoli $(1959,1961)$ with motivations coming from genetic studies. Indeed, in this example, the universe is made of a dominant and a recessive allele $\{A, a\}$ and the granules are all the possible pairs $A A, A a \equiv a A, a a$, where the orthopair is given by $(A A, a a)$ and the order relation is $a a \leq\{A a, a A\} \leq A A$, generating the structure in Fig. 1.

Andreoli also introduces the typical operations of "refinement" and "attenuation" on levels, which enable to zoom-in or zoom-out from one level to the other. As can be seen in Fig. 1, a Boolean algebra is generated by an allele, and then it is investigated as a three-valued entity using levels. This idea can be generalized to generic Boolean algebras and Andreoli himself sketches some possible ways to do this.

\section{Aggregation of orthopairs}

The order relations given in the previous section enable us to define lattice or semilattice structures. We will give these operations at first, then several possibilities to define a negation and a difference will be investigated.

We notice that we are not dealing here with an algebraic approach to these operations (such as Cattaneo et al. 2011; Moraschini 2014), nor to an abstraction of orthopairs to Boolean, or even more general, algebras. For some hint on this topic see Ciucci (2011) and [Pagliani (2008), Frame 10.11].

\subsection{Meet and join by total orderings}

The three total order relations given in Sect. 3.1 define three lattice structures and hence three different meet and join operations. Once translated on three values, we can see that they correspond to strong Kleene conjunction and disjunction (from $\preceq_{t}$ ), weak Kleene conjunction and disjunction (respectively, the min from $\preceq_{P}$ and $\preceq_{N}$ ), the Sobocinski conjunction and disjunction (respectively, the 
$\max$ from $\preceq_{N}$ and $\preceq_{P}$ ). These three pairs of operators read on orthopairs as follows.

- the strong Kleene conjunction and disjunction (Kleene 1952):

$$
\begin{aligned}
& \left(P_{1}, N_{1}\right) \sqcap\left(P_{2}, N_{2}\right):=\left(P_{1} \cap P_{2}, N_{1} \cup N_{2}\right) \\
& \left(P_{1}, N_{1}\right) \sqcup\left(P_{2}, N_{2}\right):=\left(P_{1} \cup P_{2}, N_{1} \cap N_{2}\right)
\end{aligned}
$$

This is also the standard way to define intersection and union in rough set settings, they turn out to be mathematically well-defined, even if we can have difficulties in interpreting them (see Ciucci and Dubois 2014 for a discussion).

- the weak Kleene meet and join (Kleene 1952):

$$
\begin{aligned}
& \left(P_{1}, N_{1}\right) \sqcap_{K}\left(P_{2}, N_{2}\right):=\left(P_{1}, N_{1}\right) \sqcap_{N}\left(P_{2}, N_{2}\right) \\
& \left.:=\left(\left(P_{1} \cap P_{2}\right) \cup\left[\left(P_{1} \cap N_{2}\right) \cup\left(P_{2} \cap N_{1}\right)\right], N_{1} \cap N_{2}\right)\right)
\end{aligned}
$$

$$
\begin{aligned}
& \left(P_{1}, N_{1}\right) \sqcup_{K}\left(P_{2}, N_{2}\right):=\left(P_{1}, N_{1}\right) \sqcap_{P}\left(P_{2}, N_{2}\right) \\
& :=\left(P_{1} \cap P_{2},\left(N_{1} \cap N_{2}\right) \cup\left[\left(N_{1} \cap P_{2}\right) \cup\left(N_{2} \cap P_{1}\right)\right]\right)
\end{aligned}
$$

In three-valued logics these operations make sense when the third value is interpreted as undefined. Though $\sqcup_{K}$ could look more like a conjunction than a disjunction, its real nature becomes evident once translated in three-values Ciucci et al. (2014).

- the Sobociński operations (Sobocinski 1952):

$$
\begin{aligned}
\left(P_{1}, N_{1}\right) \sqcap_{S}\left(P_{2}, N_{2}\right) & :=\left(P_{1}, N_{1}\right) \sqcup_{N}\left(P_{2}, N_{2}\right) \\
& =\left(P_{1} \backslash N_{2} \cup P_{2} \backslash N_{1}, N_{1} \cup N_{2}\right) \\
\left(P_{1}, N_{1}\right) \sqcup_{S}\left(P_{2}, N_{2}\right) & :=\left(P_{1}, N_{1}\right) \sqcup_{P}\left(P_{2}, N_{2}\right) \\
& =\left(P_{1} \cup P_{2}, N_{1} \backslash P_{2} \cup N_{2} \backslash P_{1}\right)
\end{aligned}
$$

On three values they correspond to uninorms with neutral element $\frac{1}{2}$ (Grabisch et al. 2009) and are used in conditional events to fuse conditionals (Dubois and Prade 1994), where they are known as quasi conjunction and quasi disjunction.

\subsection{Meet by partial orders}

The information ordering defines the following meet operation:

$$
\left(P_{1}, N_{1}\right) \sqcap_{I}\left(P_{2}, N_{2}\right):=\left(P_{1} \cap P_{2}, N_{1} \cap N_{2}\right)
$$

It is also known as the pessimistic combination operator in the context of belief aggregation (Lawry and Dubois 2012), since it keeps only what both orthopairs agree on, both in the positive and negative side. On three values, it corresponds to a nullnorm, more specifically the meet of $x, y$ is the median of $\left\{x, y, \frac{1}{2}\right\}$ (Grabisch et al. 2009). The corresponding join (that does not always give an orthopair) is also named the optimistic combination operator (Lawry and Dubois 2012):

$\left(P_{1}, N_{1}\right) \sqcup_{I}\left(P_{2}, N_{2}\right):=\left(P_{1} \cup P_{2}, N_{1} \cup N_{2}\right)$

and it makes sense whenever the two orthopairs are consistent, i.e., $P_{1} \cap N_{2}=\emptyset$ and $P_{2} \cap N_{1}=\emptyset$.

The other two orderings just keep one of the two side of the above meet (13) and add elements to the other side:

$$
\begin{aligned}
& \left(P_{1} \cap P_{2}, N_{1} \cup N_{2} \cup\left(P_{1} \cap B n d_{2}\right) \cup\left(P_{2} \cap B_{n} d_{1}\right)\right) \\
& \left(P_{1} \cup P_{2} \cup\left(N_{1} \cap B n d_{2}\right) \cup\left(N_{2} \cap B n d_{1}\right), N_{1} \cap N_{2}\right)
\end{aligned}
$$

As can be seen the second equation defines a disjunction more than a conjunction. In both operations, we notice that the boundary is obtained as the intersection of the two starting boundaries: $B n d_{O_{1} \sqcap O_{2}}=B n d_{1} \cap B n d_{2}$. Once interpreted on three values they both originate non-monotonic operations.

\subsection{Negations}

In three valued logics there are three different negations that extend the classical ones: they obviously differ only on the value assigned to the negation of the third value, giving rise to the standard (involutive) negation, the intuitionistic and paraconsistent negations. The value assigned to the third value $\frac{1}{2}$ is, respectively, $\frac{1}{2}, 0$ and 1 . On orthopairs, these three negations read as:

$$
\begin{aligned}
\neg(P, N) & :=(N, P) \\
\sim(P, N) & :=\left(N, N^{c}\right) \\
-(P, N) & :=\left(P^{c}, P\right)
\end{aligned}
$$

We can see that the involutive negation switches the positive and negative parts, whereas the other two just consider one side of the switching and then construct an orthopair with no boundary.

We can play with the other two total orders as we did with the standard one and obtain six other different negations. That is, in the case of order $\preceq_{N}$ we set $\frac{1}{2}$ and 0 to be the negation one of the other and then assign to the negation of 1 one of the three possible values. Similarly, for $\preceq_{P}$, $\frac{1}{2}$ and 1 will be the negation one of the other and in case of 0 one of the three values is considered as its negation. On orthopairs these six negations are:

$$
\begin{aligned}
\neg_{N}(P, N) & :=(P, \text { Bnd }) \\
\sim_{N}(P, N) & :=(\emptyset, \text { Bnd }) \\
-_{N}(P, N) & :=(\emptyset, N)
\end{aligned}
$$


Though they can appear strange at a first sight, at least some of them have a meaningful interpretation and have been studied by some authors. The negation $\neg_{N}$ is considered in Fadini (1962a), as one of the possible ways to negate a positive-boundary pair ("complex class" in Fadini's terminology). It consists in negating only the boundary that is switched with the negative part, thus moving from a positive-negative to a positive-boundary pair. Fadini also considered the three negations arising from the order $\preceq_{P}$. The involutive one $\neg_{P}$ is obtained by admitting that a negation of the Boolean and Boundary part could contain only elements outside the Boolean/Boundary parts and not all and only as it was in his other negations. Hence, the swapping of the Positive and Boundary sets. The intuitionistic and paraconsistent like negations are obtained by negation of both the Boolean and Boundary part. In these two negations, nothing is false, the difference lies in the positive part, which consists in one case in the boundary and in the other in the union of boundary and negative. We also remark that on three values, the negation $-_{P}$ corresponds to the complete negation introduced by Reichenbach in his studies on quantum mechanics (Reichenbach 1954).

As a last and different approach, let us consider an operation which is not well defined on the collection of orthopairs, in the sense that the result is a pair of sets not necessarily disjoint. The negation of $(P, N)$ consists in negating both the positive and negative part $\left(P^{c}, N^{c}\right)$ and it was introduced in Andreoli (1959). Clearly, unless the boundary is empty this is no more an orthopair, but a generic pair of sets, which can make sense in a more general context (see Sect. 5.1). This operation is introduced under a decision theoretic interpretation of an orthopair where $P$ corresponds to accept, $N$ to reject and the boundary to undecided. So, the negation of undecided is just decided which can mean either accept or reject. We will go back to this issue while discussing the connections with three-way decision theory in Sect. 6.2.

\subsection{Difference}

Also in case of the difference, several definitions are possible. Gentilhomme (1968) introduces three kinds of difference but he considers as correct one only the following:

$$
O_{1} \backslash O_{2}:=O_{1} \sqcap \neg O_{2}=\left(P_{1} \cap N_{2}, N_{1} \cup P_{2}\right)
$$

The justification of considering this operation as a difference is more evident if we consider it as a nested pair, indeed, it would be written as $\left(P_{1}, U_{1}\right) \backslash\left(P_{2}, U_{2}\right)=$ $\left(P_{1} \backslash U_{2}, U_{1} \backslash P_{2}\right)$. The other two differences can be obtained similarly to the previous one, but using a different negation:

$$
\begin{aligned}
& O_{1} \sqcap \sim O_{2}=\left(P_{1} \cap N_{2}, N_{1} \cup N_{2}^{c}\right) \\
& O_{1} \sqcap-O_{2}=\left(P_{1} \cap P_{2}^{c}, N_{1} \cup P_{2}\right)
\end{aligned}
$$

Again, once interpreted on nested pairs, their meaning becomes more evident. The first one $\left(P_{1} \backslash U_{2}, U_{1} \backslash U_{2}\right)$ corresponds to accepting no risk, while the second $\left(P_{1} \backslash P_{2}, U_{1} \backslash P_{2}\right)$ in accepting the maximum of the risk, since the positive and boundary region are larger than the first one.

In Lawry and Tang (2012), we can find an operation that sounds to be a more natural difference in the orthopair context:

$O_{1} \ominus O_{2}:=\left(P_{1} \backslash N_{2}, N_{1} \backslash P_{2}\right)$

By removing from the positive (negative) part of $O_{1}$ what $\mathrm{O}_{2}$ considers as negative (positive), we make $O_{1}$ consistent with $\mathrm{O}_{2}$. Indeed, this difference is at the basis of the consensus operation (Lawry and Dubois 2012)

$$
O_{1} \odot O_{2}:=\left(P_{1} \backslash N_{2} \cup P_{2} \backslash N_{1}, N_{1} \backslash P_{2} \cup N_{2} \backslash P_{1}\right)
$$

in which two orthopairs are merged in a consistent way, by eliminating, through the difference operator, the discrepancies between the two orthopairs. Indeed, it can be seen that the following holds:

$$
O_{1} \odot O_{2}=\left(O_{1} \ominus O_{2}\right) \sqcup_{I}\left(O_{2} \ominus O_{1}\right) .
$$

Remark 2 All the operations outlined in this section can be used to aggregate orthopairs with different aims according to the operation and to the application. Moreover, they can be used in two ways to combine orthopairs: inside the same granulation or between two different granulations. Some examples are:

- if two orthopairs represent two agents opinion on the same fact, then we can compute their difference through $\ominus$ and reach a consensus between them using the operator $\odot$;

- similarly, two agents point of view can be combined in a pessimistic or optimistic way, using the operations $\sqcap_{I}, \sqcup_{I}$;

- Sobocinski operations are standard conjunction and disjunction operations on conditional events;

- if we want to aggregate two shadowed sets, then a first choice is to use Kleene lattice operations, that corresponds to min and max on fuzzy sets;

- in case of decision theory, where the regions of the orthopair represent accept and reject, operations can be used to aggregate two different decisions on the same subject (see Sect. 6.2 and future works in Sect. 7.2). 


\section{Generalizations}

We now introduce three different generalizations of orthopairs that can represent different kinds of knowledge.

\subsection{Paraconsistent pairs}

A first straightforward generalization can be obtained by dropping the (only) condition of an orthopair: $P \cap N=\emptyset$. In this way we just get a pair of subsets of the universe $(A, B)$. Keeping the same interpretation of these two sets as in orthopairs, we have that the intersection $F \cap G$ represents the objects on which we have contrasting information, for instance given by two agents. In this sense, a pair of this kind is linked to (partial) paraconsistent logic. More precisely, it is closely related to Belnap's four valued logic Belnap (1977). Indeed, as orthopairs are in bijection with three-valued sets, paraconsistent pairs are in bijection with four-valued sets. Let $h: X \mapsto\{0,1, u, c\} \quad$ ( $u$ stands for unknown, $c$ for contradictory), then

$$
\begin{array}{rll}
x \in A \backslash B & \text { iff } & \mathrm{h}(\mathrm{x})=1 \\
x \in B \backslash A & \text { iff } & \mathrm{h}(\mathrm{x})=0 \\
x \in A \cap B & \text { iff } & \mathrm{h}(\mathrm{x})=\mathrm{c} \\
x \in(A \cup B)^{c} & \text { iff } & \mathrm{h}(\mathrm{x})=\mathrm{u}
\end{array}
$$

A bi-lattice structure Fitting (1989) can be defined on these values whose orderings are the truth ordering (1), $0 \leq\{u, c\} \leq 1$, and the information ordering (7), $u \leq\{0,1\} \leq c$. A paraconsistent pair can be originated, as already said, by two different agents whose knowledge can be represented by an orthopair. Indeed, given two orthopairs $\left(P_{1}, N_{1}\right)$ and $\left(P_{2}, N_{2}\right)$ we can define a paraconsistent pair as $\left(P_{1} \cup P_{2}, N_{1} \cup N_{2}\right)$, whose contradictory region is clearly given by $\left(P_{1} \cap N_{2}\right) \cup\left(P_{2} \cap N_{1}\right)$.

\subsection{Possibility theory}

Possibility theory is an uncertainty theory to manage missing information. It has a bipolar flavor, since it can manage separately positive and negative information (Dubois and Prade 2015a); Dubois et al. 2001). This points directly to orthopairs, which share the same spirit. Possibility distributions and measure however have a greater flexibility in representing information. As we are going to explain orthopairs are just particular possibility distributions, which can thus be seen as a generalization of orthopairs.

Definition 4 A possibility distribution on a set $S$ is a mapping: $\pi: S \mapsto[0,1]$. It is said Boolean if only the values 0,1 are admitted: $\pi: S \mapsto\{0,1\}$.
In our case, given a set of propositional variables $\mathcal{A}$, we consider the collection of valuations that we can define on $\mathcal{A}: \Omega=\{\omega: \mathcal{A} \mapsto\{0,1\}\}$ and we are interested on Boolean possibility distribution defined on $\Omega$.

The possibility distribution attached to an orthopair of variables $(P, N)$ is the characteristic function of the set of models of the formula $\phi=\left[\bigwedge_{a \in P} a \wedge \bigwedge_{a \in N} \neg a\right]$. That is

$\pi_{(P, N)}(\omega):= \begin{cases}1 & \text { if } \omega \models\left[\bigwedge_{\mathrm{a} \in \mathrm{P}} \mathrm{a} \wedge \bigwedge_{\mathrm{a} \in \mathrm{N}} \neg \mathrm{a}\right] \\ 0 & \text { otherwise }\end{cases}$

Clearly, not all possibility distributions, nor even if we restrict to the Boolean ones can be represented by an orthopair. It is thus evident that orthopairs can only generate a subset of Boolean possibility distribution. In particular, those that take an hyper-rectangle shape on the space $\{0,1\}^{n}$, i.e., a Cartesian product of subsets of $\{a, \neg a\}$. To capture any Boolean possibility distribution a set of orthopairs is needed Ciucci et al. (2014).

\subsection{Atanassov intuitionistic fuzzy sets}

If we want to generalize orthopairs to a fuzzy environment, we need to give a definition of orthogonality on fuzzy sets corresponding to the Boolean requirement $P \cap N=\emptyset$. If we express this condition in terms of characteristic functions, we can see that it corresponds to ask that for all $x$, $\chi_{P}(x)+\chi_{N}(x) \leq 1$, i.e., all elements $x$ can belong at most to one between $P$ and $N\left(\chi_{A}\right.$ is the characteristic function of the set $A$ ). This last relation can be immediately generalized to obtain an orthogonality relation on fuzzy membership functions $f, g$ (Cattaneo and Ciucci 2006; Cattaneo and Manià 1974):

$f(x)+g(x) \leq 1$.

This condition exactly characterizes Atanassov intuitionistic fuzzy sets (IFS) (Atanassov 1986, 1999, 2012), a very well known tool to represent vague bipolar information. It has many applications in information retrieval, data mining, decision making, etc. Clearly, IFS have a greater flexibility in knowledge representation than orthopairs, that also results in a wider possibility to define operations on IFS with respect to the ones on orthopairs introduced in Sect. 4. For an overview see the book (Atanassov 2012).

We remark that Çoker in Çoker (1996) introduced the notion of "intuitionistic set" as the Boolean counterpart of IFS, of course they coincide with orthopairs. We also underline that the term "intuitionistic" has been strongly criticized in this context, since IFS have no relation with (standard) intuitionistic logic (Dubois 2005; Cattaneo and Ciucci 2006). 


\section{Related paradigms based on a tri-partition of the universe}

Some knowledge representation and reasoning tools deals with a tri-partition of a universe and as such can be easily put in correspondence with orthopairs. In this section, we put forward these connections.

\subsection{Hexagon of opposition}

An opposition is a relation between two logical statements expressing an opposite point of view. The study on oppositions starts in ancient Greece and has its main result is the Square of Opposition by Aristotle. In the last years, we can assist to a renewal of interest around this topic, mainly due by Jean-Yves Béziau Béziau (2003, 2012). Several generalizations of the traditional square are defined, adding new kind of oppositions and thus different geometrical representations: cube, hexagon, tetrahedron, etc. (Reichenbach 1952; Hacker 1975; Dubois and Prade 2012a; Demey 2012; Smessaert and Demey 2014; Dekker 2015) or generalization of the underlying logic from Boolean to many-valued (Murinová and Novák 2013, 2014; Dubois and Prade 2015b; Ciucci et al. 2016).

Here we are interested in the hexagon of opposition, as proposed by Blanché Blanché (1953, 1966), that to the traditional square adds two other vertices. As explained in Dubois and Prade (2012a), a hexagon of opposition can be obtained by any tri-partition of a universe, hence by any orthopair.

Given an orthopair $(P, N)$, the six vertices of the hexagon are: $P, N, B n d, U p p, P \cup N, P^{c}$ linked as in Fig. 2.

The different links between vertices represent different kinds of relation among them: $P, N, B n d$ are mutually contrary; $P, P^{c}$ and $N, U p p$ are contradictory; arrows indicate sub-altern relationship and double-lines a subcontrary one. In any hexagon we can find three different square of opposition, in this case they are: $\left(P, U p p, N, P^{c}\right)$, $\left(P, B n d, P^{c}, P \cup N\right)$ and $(U p p, B n d, N, P \cup N)$. We notice that the top element $P \cup N$ represents the objects on which

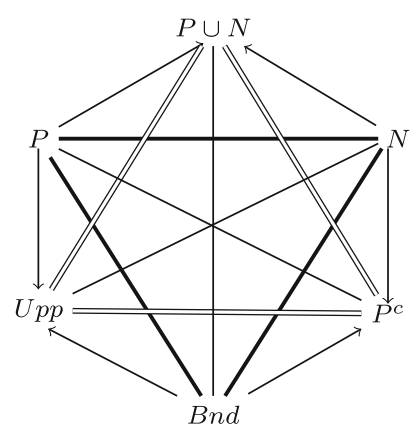

Fig. 2 Hexagon induced by an orthopair we are certain, either in a positive or negative sense, whereas the bottom vertex is the collection on unknown objects.

Oppositions can be found at work in several knowledge representation tools, such as formal concept analysis, possibility theory, abstract argumentation, rough set theory or analogical proportions (Dubois and Prade 2012a; Amgoud and Prade 2013; Ciucci et al. 2014; Miclet and Prade 2014). Thus, the link with the hexagon of opposition could also open the possibility to connect orthopairs with all these paradigms.

\subsection{Three-way decisions}

A tri-partition of the universe is also at the basis of threeway decision (3WD) theory. Starting from the rough set division of the universe in three regions, Yao defined this new paradigm of decision theory (Yao 2009b), where besides accept and reject also a deferment of the decision is possible. A three-way decision procedure consists, at high level, in two steps: trisecting the universe and then acting, i.e., taking a different strategy on objects belonging to different regions (Yao 2015). Thus, orthopairs deal mainly with the first step, and the methods given in Sect. 2 could potentially also be useful in three-way decision (of course rough sets are a standard tool in 3WD). Moreover, as already said, one of the possible interpretations of the three regions of an orthopair is exactly the decision theoretic one and this interpretation has sometimes explicitly been taken into account while studying orthopairs. In Sect. 4.3, an operator was introduced that changes a decision from decided to undecided. According to Andreoli, who introduced such an operation, this is the first step of a procedure to classify objects. At first we divide them between decided and undecided and then the decided ones are classified as chosen or rejected. This procedure works in the opposite direction with respect to the three-way sequential decision theory introduced in Yao (2013), which at first classifies objects on which we are sure and then in following steps classifies the other ones (see Fig. 3).

Other operations introduced on orthopairs could turn out to be useful in $3 \mathrm{WD}$, for instance to aggregate different tripartitions arising from different agents/procedures. For instance, the reference to "risk" used by Gentilhomme while introducing the two differences (18) directly points to $3 \mathrm{WD}$. Moreover, it is not difficult to image a possibility to merge two tri-partitions to reach a consensus between

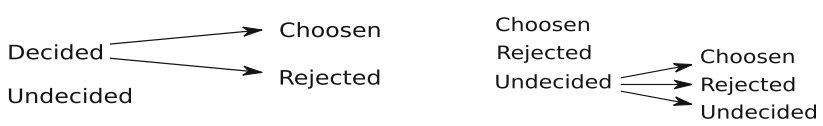

Fig. 3 Sequential decision making (Andreoli left, Yao right) 
them or simply by a (more or less conservative) conjunction.

On the other hand all the links between orthopairs and other paradigms outlined is this section and in the previous one could be related also to $3 \mathrm{WD}$ with the possibility to make them interact.

\subsection{Version spaces}

Version spaces is an approach to learning by examples (Mitchell 1982). The training set is divided in positive and negative examples with a consistency requirement: no instances conflict. In our language, an orthopair. Then the aim, is to build a classifier, that matches positive instances and does not accept negative ones. The search space for such a classifier is delimited by two sets: the most specific and the most general possible ones given the training set. This strategy requires an order relation among classifiers:

A pattern $P 1$ is more specific than or equal to pattern $P 2$

\section{$(P 1 \geq P 2)$ if and only if pattern $P 1$ matches a subset of all instances which $P 2$ matches}

For instance a classifier that accepts "red and round object" is more specific than a classifier that accepts "red objects". Hence, the first one will have less positive examples and more negative examples than the second one. That is the truth ordering $\preceq_{t}$ on orthopairs.

If we identify a classifier with the instances it represents, then, it can be seen as an orthopair and hence a version space is a collection of orthopairs. This points to possibility theory, since, as explained in Sect. 5.2, a possibility distribution can be described by a collection of orthopairs. Indeed, connections between version spaces and possibility theory have already been established Prade and Serrurier (2008).

\section{Conclusions and open problems}

Orthopairs are a simple description of bipolar information, which are at the basis of many representation knowledge and reasoning tools. We have here framed them into a granular computing approach by seeing an orthopair as a granule and the order between granules as a possibility to generate a hierarchy. Several connections with other paradigms have been also laid bare. Yet, all these connections are still to be fully understood. Indeed, several questions and possible research directions remain open, as exemplified in the following.

\subsection{Open problems on orthopairs}

- Study the relations and dependencies among the different granular structures generated by the different orderings, similarly as was done in Yao et al. (2012) for standard granules.

- Uncertainty measures. If an orthopair is aimed to represent uncertainty, than it should be important to measure to which amount our knowledge is certain or not. In case of shadowed sets outlined in Sect. 2.2, this measure is intrinsic to their definition, but this is not always the case and we can think to several approaches according to the application domain. For instance, we could measure how many objects are in the boundary with respect to the universe and then study how this uncertainty propagates along the granular structure (i.e., by aggregating orthopairs). In presence of a collection of orthopairs (as in version spaces or in rough set theory), overall measure could also be fruitful to characterize the whole system.

- Clarify the algebraic picture and define orthopairs and related operations on more abstract settings. Some results in this sense are already known, we have algebraic approaches to orthopairs (Cattaneo et al. 2011) and also of pair of nested/ortho pairs defined on Boolean or Heyting algebras (Monteiro 1980; Vakarelov 1977; Walker 1994). For more details see Ciucci (2011) and [Pagliani (2008), Frame 10.11].

\subsection{Open problems related to other paradigms}

- Partial orthopairs. We can think of another generalization of orthopairs, where some objects are undefined, i.e., out of the scope of the investigation described by the orthopair. This leads to a partition in four regions of the universe, but with a different interpretation with respect to the paraconsistent one. An example of this granulation can be obtained by rough sets based on partial coverings Csajbók (2013).

- The role of Formal Concept Analysis (FCA) in this picture has to be clarified. Indeed, besides the already mentioned possibility to define a hexagon of opposition on FCA, there are several other links with the paradigms presented here. Indeed, version spaces can be described in terms of formal concept analysis (Ganter and Kuznetsov 2003), FCA has been generalized by ideas from possibility theory (Dubois and Prade 2012b) and, recently, orthopairs have been explicitly used to generalize FCA (Qi et al. 2014).

- As anticipated in Remark 2 and in Sect. 6.2, the different aggregation operations outlined on orthopairs 
can be thought to be put at work on the tri-partition of 3WD. For instance to fuse decisions taken from different agents on the same subject.

\section{References}

Aguirre F, Destercke S, Dubois D, Sallak M, Jacob C (2014) Inclusion-exclusion principle for belief functions. Int J Approx Reason 55(8): 1708-1727

Amgoud L, Prade H (2013) A formal concept view of abstract argumentation. In: van der Gaag LC (ed) Proceedings 12th Eur. Conf. Symb. and Quant. Appr. to Reas. with Uncert. (ECSQARU'13), Utrecht, July 8-10, LNCS 7958, pp 1-12. Springer Andreoli G (1959) Algebre di boole - algebre di insieme - algebre di livelli. Giornale di Matematiche di Battaglini vol LXXXVII, pp 3-22

Andreoli G (1961) Dicotomie e tricotomie (anelli booleani triadici ed algebre booleane a tre valori). Ricerca 11:1-10

Atanassov KT (1986) Intuitionistic fuzzy sets. Fuzzy Sets Syst 20:87-96

Atanassov KT (1999) Intuitionistic fuzzy sets, vol 35. Physica Verlag, Springer, Heidelberg

Atanassov KT (2012) On intuitionistic fuzzy sets theory, studies in fuzziness and soft computing, vol 283, Springer, Heidelberg

Belnap ND (1977) A useful four-valued logic. In: Dunn JM, Epstein G (eds) Modern uses of multiple-valued logic, D. Reidel Publishing Company, Dordrecht, pp 8-37

Béziau JY (2003) New light on the square of oppositions and its nameless corner. Log Investig 10:218-233

Béziau JY (2012) The power of the hexagon. Log Univ 6(1-2):1-43

Blamey S (1985) Partial logic. In: Gabbay DM, Guenthner F (eds) Handbook of philosophical logic, vol. 3, p 1-70. D. Reidel Publishing Company, Dordrecht

Blanché R (1953) Sur l'opposition des concepts. Theoria 19:89-130

Blanché R (1966) Structures intellectuelles. Essai sur l'Organisation Systématique des Concepts. Vrin, Paris

Cattaneo G, Ciucci D (2006) Basic intuitionistic principles in fuzzy set theories and its extensions (a terminological debate on Atanassov IFS). Fuzzy Sets Syst 157:3198-3219

Cattaneo G, Ciucci D, Dubois D (2011) Algebraic models of deviant modal operators based on de morgan and kleene lattices. Inf Sci 181(19):4075-4100

Cattaneo G, Manià A (1974) Abstract orthogonality and orthocomplementation. Proc Camb Philos Soc. 76:115-132

Ciucci D (2011) Orthopairs: a simple and widely used way to model uncertainty. Fundam Inform 108(3-4):287-304

Ciucci D (2014) Orthopairs in the 1960s: historical remarks and new ideas. In: Cornelis C, Kryszkiewicz M, Slezak D, Ruiz EM, Bello R, Shang L (eds) Proc RSCTC, LNCS, vol 8536, Cham

Ciucci D, Dubois D (2014) Three-valued logics, uncertainty management and rough sets. Trans Rough Sets 17:1-32

Ciucci D, Dubois D, Lawry J (2014) Borderline vs. unknown: comparing three-valued representations of imperfect information. Int J Approx Reason 55(9): 1866-1889

Ciucci D, Dubois D, Prade H (2014) The structure of oppositions in rough set theory and formal concept analysis-toward a new bridge between the two settings. In: Beierle C, Meghini C (eds) Proceedings of the 8th international symposium on foundations of information and knowledge systems (FoIKS'14), Bordeaux, Mar. 3-7, LNCS, vol 8367. Springer, Cham, pp 154-173

Ciucci D, Dubois D, Prade P (2016) Structures of opposition induced by relations. Ann Math Artif Intell. doi:10.1007/s10472-015$9480-8$
Çoker D (1996) A note on intuitionistic sets and intuitionistic points. Turk J Math 20:343-351

Crosscombe M, Lawry J (2015) Exploiting vagueness for multi-agent consensus. In: Bai $\mathrm{Q}$, Ren $\mathrm{F}$, Zhang $\mathrm{M}$, Ito $\mathrm{T}$ (eds) The Proceedings of the 2nd International Workshop on Smart Simulation and Modelling for Complex Systems at IJCAI 2015

Csajbók Z (2013) Approximation of sets based on partial covering. Trans Rough Sets 16:144-220

Dekker PJ (2015) Not only barbara. J Logic Lang Inf 24(2):95-129

Demey L (2012) Algebraic aspects of duality diagrams. In: Cox PT, Plimmer B, Rodgers PJ (eds) Proceedings of the 7th International Conference on Diagrammatic Representation and Inference (Diagrams'12), Canterbury, July 2-6, LNCS, vol 7352. Springer, Heidelberg, pp 300-302

Dubois D, Gottwald S, Hajek P, Kacprzyk J, Prade H (2005) Terminological difficulties in fuzzy set theory-the case of Intuitionistic Fuzzy Sets. Fuzzy Sets Syst 156:485-491

Dubois D, Prade H (1994) Conditional objects as nonmonotonic consequence relationships. IEEE Trans Syst Man Cybern 24(12):1724-1740

Dubois D, Prade H (2012a) From Blanché's hexagonal organization of concepts to formal concept analysis and possibility theory. Log Univ 6:149-169

Dubois D, Prade H (2012b) Possibility theory and formal concept analysis: characterizing independent sub-contexts. Fuzzy Sets Syst 196:4-16

Dubois D, Prade H (2015a) Possibility theory and its applications: where do we stand? In: Kacprzyk J, Pedrycz W (eds) Springer Handbook of Computational Intelligence, Springer, Dordrecht, pp 31-60

Dubois D, Prade H (2015b) Gradual structures of oppositions. In: Esteva F, Magdalena L, Verdegay JL (eds) Enric Trillas: passion for fuzzy sets, studies in fuzziness and soft computing, vol 322. Springer, Cham, pp 79-91

Dubois D, Prade H, Smets P (2001) "Not impossible" vs. "guaranteed possible" in fusion and revision. In: Benferhat $\mathrm{S}$, Besnard $\mathrm{P}$ (eds) Symbolic and quantitative approaches to reasoning with uncertainty, 6th European conference, ECSQARU 2001, Toulouse, France, September 19-21, 2001, Proceedings, LNCS, vol 2143. Springer, Berlin, London, pp 522-531

Fadini A (1962) Il calcolo delle classi in una logica a tre valori di verità. Giornale di Matematiche di Battaglini vol XC, pp 72-91

Fadini A (1962) Teoria degli elementi complessi nelle Algebre di Boole. Ann del Pontif Ist Super di Sci e Lett S. Chiara $12: 223-243$

Fitting M (1989) Bilattices and the theory of truth. J Philos Logic $18: 225-256$

Ganter B, Kuznetsov SO (2003) Hypotheses and version spaces. In: de Moor A, Lex W, Ganter B (eds) Conceptual structures for knowledge creation and communication, 11th International Conference on Conceptual Structures, ICCS 2003 Dresden, Germany, July 21-25, 2003 Proceedings, LNCS, 2746:83-95

Gentilhomme MY (1968) Les ensembles flous en linguistique. Cah de Linguist Theor et Appl Bucar 47:47-65

Grabisch M, Marichal JL, Mesiar R, Pap E (2009) Aggregation functions. Cambridge University Press, New York

Hacker E (1975) The octagon of opposition. Notre Dame J Form Logic 16:352-353

Kacprzyk J, Pedrycz W (eds) (2015) Springer handbook of computational intelligence, Springer, Dordrecht

Kleene SC (1952) Introduction to metamathematics. North-Holland Pub. Co., Amsterdam

Lawry J, Dubois D (2012) A bipolar framework for combining beliefs about vague propositions. In: Brewka G, Eiter T, McIlraith SA (eds) Proceedings of the thirteenth international conference on 
principles of knowledge representation and reasoning (KR 2012), Roma, Italy, pp 530-540

Lawry J, González Rodríguez I (2011) A bipolar model of assertability and belief. Int J Approx Reason 52(1):76-91

Lawry J, Tang Y (2012) On truth-gaps, bipolar belief and the assertability of vague propositions. Artif Intell 191-192:20-41

Miclet L, Prade H (2014) Analogical proportions and square of oppositions. In: Laurent A et al. (eds) Proceedings of the 15th international conference on information processing and management of uncertainty in knowledge-based systems, July 15-19, Montpellier, CCIS, vol 443. Springer, Cham, pp 324-334

Mitchell T (1982) Generalization as search. Artif Intell 18:203-226

Monteiro A (1980) Sur les algèbres de Heyting symétriques. Port Math 39:1-237

Moraschini T (2014) An algebraic study of exactness in partial contexts. Int J Approx Reason 55(1):457-468

Murinová P, Novák V (2013) The analysis of the generalized square of opposition. In: Montero J, Pasi G, Ciucci D (eds) Proceedings of the 8th conference of the european society for fuzzy logic and technology (EUSFLAT'13), Milano, September 11-13. Atlantis Press

Murinová P, Novák V (2014) Analysis of generalized square of opposition with intermediate quantifiers. Fuzzy Sets Syst 242:89-113

Pagliani P, Chakraborty M (2008) A geometry of approximation, Springer, Dordrecht

Pawlak Z (1982) Rough sets. Int J Comp Inf Sci 11:341-356

Pedrycz W (1998) Shadowed sets: representing and processing fuzzy sets. IEEE Trans Syst Man Cybern Part B Cybern 28(1):103-109

Pedrycz W (2005) Granular computing with shadowed sets. Springer, Berlin 23-32

Pedrycz W (2009) From fuzzy sets to shadowed sets: interpretation and computing. Int J Intell Syst 24:48-61

Pedrycz W, Vukovich G (2002) Granular computing with shadowed sets. Int J Intell Syst 17:173-197

Prade H, Serrurier M (2008) Bipolar version space learning. Int J Intell Syst 23:1135-1152

Qi J, Wei L, Yao Y (2014) Three-way formal concept analysis. In: Miao D, Pedrycz W, Slezak D, Peters G, Hu Q, Wang R (eds) Rough sets and knowledge technology - 9th International Conference, RSKT 2014, Shanghai, China, October 24-26, 2014, Proceedings, LNCS, 8818:732-741

Reichenbach H (1952) The syllogism revised. Philos Sci 19(1):1-16

Reichenbach H (1954) Philosophic foundations of quantum mechanics. University of California Press, Berkeley

Shapiro S (2006) Vagueness in context. Oxford University Press, Oxford
Smessaert H, Demey L (2014) Logical geometries and information in the square of oppositions. J Logic Lang Inf 23(4):527-565

Sobocinski B (1952) Axiomatization of a partial system of threevalue calculus of propositions. J Comp Syst 1:23-55

Vakarelov D (1977) Notes on n-lattices and constructive logic with strong negation. Stud $\log$ 36:109-125

Vetterlein $T$ (2015) Logic of prototypes and counterexamples: possibilities and limits. In: The Proceedings of 16th World Congress of the International Fuzzy Systems Association (IFSA), p 697-704

Walker E (1994) Stone algebras, conditional events, and three valued logic. IEEE Trans Syst Man Cybern 24(12):1699-1707

Wu J, Chiclana F, Herrera-Viedma E (2015) Trust based consensus model for social network in an incomplete linguistic information context. Appl Soft Comp 35:827-839

Yao JT, Vasilakos AV, Pedrycz W (2013) Granular computing: perspectives and challenges. IEEE Trans Cybern 43(6): 1977-1989

Yao Y (1993) Interval-set algebra for qualitative knowledge representation. In: Proceedings of the Fifth international Conference on Computing and Information p 370-374

Yao YY (1998) Constructive and algebraic methods of the theory of rough sets. J Inf Sci 109:21-47

Yao Y (2009a) Interval sets and interval-set algebras. In: Proceedings of the 8th IEEE international conference on cognitive informatics pp 307-314

Yao Y (2009b) Three-way decision: an interpretation of rules in rough set theory. In: Wen P, Li Y, Polkowski L, Yao Y, Tsumoto S, Wang G (eds) Rough sets and knowledge technology, 4th International conference, RSKT 2009, Gold Coast, Australia, July 14-16, 2009. Proceedings, LNCS, vol 5589. Springer, Heidelberg, pp 642-649

Yao Y (2013) Granular computing and sequential three-way decisions. In: Lingras $\mathrm{P}$, Wolski $\mathrm{M}$, Cornelis $\mathrm{C}$, Mitra S, Wasilewski P (eds) RSKT, Lecture notes in computer science 8171:16-27

Yao Y (2015) Rough sets and three-way decisions. In: Ciucci D, Wang G, Mitra S, Wu W (eds) RSKT proceedings, LNCS, vol 9436

Yao Y, Lingras P, Wang R, Miao D (2009) Interval set cluster analysis: a re-formulation. In: Proceedings of the RSFDGrC 2009, LNCS, 5908:398-405

Yao Y, Zhang N, Miao D, Xu F (2012) Set-theoretic approaches to granular computing. Fundam Inform 115(2-3):247-264 